\title{
A Processing Method to Increase Production of Biomethane from Lignite
}

\author{
Xingcheng Zhao ${ }^{1, \text { a }}$, Bo Wang ${ }^{2, \mathrm{~b}}$, Yuhong $\mathrm{Xie}^{3, \mathrm{c}^{*}}$, and Xin Feng ${ }^{4} \mathrm{~d}^{*}$
}

${ }^{1}$ School of Environmental Science and Safety Engineering, Tianjin University of Technology, Tianjin 300384, China.

${ }^{2}$ Key Laboratory of Environmental Biotechnology, Research Center for Eco-Environmental Sciences, Chinese Academy of Sciences, Beijing 100085, China

${ }^{3}$ School of Environmental Science and Safety Engineering, Tianjin University of Technology, Tianjin 300384, China.

${ }^{4}$ School of Environmental Science and Safety Engineering, Tianjin University of Technology, Tianjin 300384, China.

aJasonZhao777@outlook.com, 'bangbo1320201006@163.com, 'xieyuhong@tjut.edu.cn, dxfeng72100@163.com

*Yuhong Xie and Xin Feng are both corresponding authors of this paper.

Keywords: methanogenesis; lignite; microbial; hydrogen peroxide

Abstract. This study evaluated the bioavailability of lignite from Shanxi Province (China) via using domestic anaerobic sludge as the source of exogenous flora. After pretreatment of lignite with hydrogen peroxide at different concentrations, the levels of soluble organic carbon and organic acid were increased significantly. hydrogen peroxide pretreatment can improve the solubility and bioavailability of lignite and influence methane production, making them viable processing options for increasing microbial production of coalbed methane.

\section{Introduction}

As a resource with abundant reserves, coal occupies more than $70 \%$ of global fossil fuels [1]. Currently, over $90 \%$ of lignite is used for conventional combustion and power generation among all of coals. However, the greenhouse gases and other harmful substances released during combustion process can cause serious environmental pollution [2]. In recent years, the process of lignite biogasification has been developed as the emerging and alternative cleaner energy source to produce methane [3]. Compared with traditional combustion methods, biogenic coalbed methane has many advantages, such as low energy consumption, moderate methane production conditions, high methane productivity efficiency, and simple equipment requirements etc. [4].

The heterogeneity and complexity of coal limit its direct use by microorganisms; therefore, without improvement of the physical, chemical, and biological pretreatment methods to enhance the solubility and bioavailability of coal, it will remain difficult to increase biomethane output in a coal seam [5]. There are two primary advantages in using hydrogen peroxide to pretreat lignite. First, the dissolution of lignite by hydrogen peroxide can produce organic acids (e.g., acetic acid) that are easily used by methanogens, which can promote the bioavailability of lignite [6]. Second, the decomposition of hydrogen peroxide produces water and oxygen without introducing harmful elements into the coal seam.

The objectives of this study were as follows: (1) to pretreat lignite with different concentrations of hydrogen peroxide; (2) to determine the total organic carbon and organic acid contents in the supernatant after reaction, and evaluate lignite solubility and methane production after the pretreatment.

\section{Materials and methods}

Sample. The coal samples were obtained from the Qiaojiawan Coal Mine.

Pretreatment of coal samples. At room temperature, $2 \mathrm{~g}$ lignite was added to $100 \mathrm{~mL}$ hydrogen peroxide solution at different concentrations $(0.5,1.5$, and $2.5 \mathrm{M})$ with triplicates, and the 
control experiment was conducted in a same mass of $2 \mathrm{~g}$ lignite added but with $100 \mathrm{~mL}$ water. After reaction for 10 days, the supernatant was filtered using a $0.45-\mu \mathrm{m}$ organic system filter membrane prior to the chemical analyses.

Setup for experiments. According to the method of Huang et al. [7], a basic culture solution, a trace element culture solution, and a vitamin solution were configured. The $\mathrm{pH}$ of the medium was adjusted to $7.0 \pm 0.2$ using sodium hydroxide. Anaerobic sludge from domestic sewage treatment plants (sourced from Bengbu Jingsheng Environmental Protection Technology Co. Ltd., Anhui, China) was used as the source of exogenous flora. After domestication for several times, the supernatant was used as the inoculum. To investigate the effects of bioavailability and solubility on methane production, the following experiments were designed. In all assays, $5 \mathrm{~mL}$ of the inoculum was added and the samples were cultured in an anaerobic incubator at $35^{\circ} \mathrm{C}$. This study referred to the method proposed by Huang et al. [8], i.e., 2 g lignite with different concentrtions of hydrogen peroxide treated was added to $25 \mathrm{~mL}$ of methanogenic nutrient solution. The concentration of the methanogenic nutrient solution used in this part was twice of the original methanogenic nutrient solution in order to keep the concentration of methanogenic nutrient solution used in all experiments consistent. Seen Table 1 for other conditions.

Table 1. Description of processing methods and conditions

Processing methods

Description

Treatment 1

Add $25 \mathrm{~mL}$ of pretreated supernatant of coal and water mixture

Treatment 2

Add $25 \mathrm{~mL}$ of supernatant pretreated with $0.5 \mathrm{M}$ hydrogen peroxide

Treatment 3

Add $25 \mathrm{~mL}$ of supernatant pretreated with $1.5 \mathrm{M}$ hydrogen peroxide

Treatment 4

Add $25 \mathrm{~mL}$ of supernatant pretreated with $2.5 \mathrm{M}$ hydrogen peroxide

Chemical analyses. A Dongxi 4100A gas chromatograph equipped with a thermal conductivity detector was used to measure the methane concentration in the bioassay. Nonpurgeable organic carbon concentration in the solution was measured with a the total organic carbon analyzer (Vario the total organic carbon, Elementar) as the dissolved organic carbon concentration. Monitoring of volatile fatty acids (formic and acetic acids) was conducted via the high-performance liquid chromatograph equipped with a $\mathrm{C} 18$ reversed-phase column $(10 \mu \mathrm{m}, 4.6 \mathrm{~mm}$ i.d. $\times 250 \mathrm{~mm})$. A phosphate buffer $(10 \mathrm{mM})$ was used as a mobile phase with a flow rate of $1 \mathrm{~mL} \mathrm{~min}{ }^{-1}$.

\section{Results and discussion}

As an oxidant, hydrogen peroxide can cause pretreated lignite to produce more microorganisms to facilitate the use of hydrophilic functional groups (e.g., ketone, hydroxyl, and carboxyl) [9]. The concentrations of the dissolved organic carbon in lignite pretreated with different concentrations of hydrogen peroxide (Treatments 2-4) and Treatment 1 are shown in Fig. 1. With the increase of hydrogen peroxide concentration, the concentration of the total organic carbon increased continuously. The concentration of the dissolved organic carbon after pretreatment with $2.5 \mathrm{M}$ hydrogen peroxide was $628.3 \mathrm{mg} \mathrm{C} \mathrm{L}^{-1}$, which was $99 \%$ and $39 \%$ higher than that of the dissolved organic carbon pretreated with 0.5 and $1.5 \mathrm{M}$ hydrogen peroxide, respectively. Compared with Treatment $4\left(1.6 \mathrm{mg} \mathrm{C} \mathrm{L}^{-1}\right)$, the the dissolved organic carbon concentration in the supernatant after treatment with hydrogen peroxide was increased significantly. In addition, more organic acids were dissolved from the lignite pretreated with hydrogen peroxide, and the organic acid concentration increased with the increase of hydrogen peroxide concentration. The concentrations of formic and acetic acids after pretreatment with $2.5 \mathrm{M}$ hydrogen peroxide were 138.2 and $101.6 \mathrm{mg} \mathrm{C} \mathrm{L}^{-1}$, respectively. No organic acid was detected in Treatment 1 . This indicated that the dissolved organic carbon and organic acids cannot be extracted from lignite using water alone. The dissolved the dissolved organic carbon and organic acids were derived via the reaction of hydrogen peroxide and lignite, which revealed that the hydrogen peroxide pretreatment method can increase the solubility 
of coal and produce substrates (formic and acetic acids) easily used by methanogens to increase the bioavailability, resulted in achieving the increase of final methane production ultimately.

Methane production continued to increase with increasing concentration of hydrogen peroxide (Fig. 1). The final yield of methane from lignite pretreated by $2.5 \mathrm{M}$ hydrogen peroxide was $193.1 \mu \mathrm{mol}$ $\mathrm{g}^{-1}$, which was $30 \%$ and $22 \%$ higher than that of lignite pretreated with 0.5 and $1.5 \mathrm{M}$ hydrogen peroxide. Treatment 1 produced the lowest amount of methane $\left(126.5 \mu \mathrm{mol} \mathrm{g}{ }^{-1}\right)$, which might be attributable to the fact that less organic matter could be dissolved from the lignite. As shown in Fig. 1 , the final methane yield was related to the concentration of organic matter dissolved from the aqueous solution. As the concentration of dissolved organic matter increased, the final methane yield also improved.

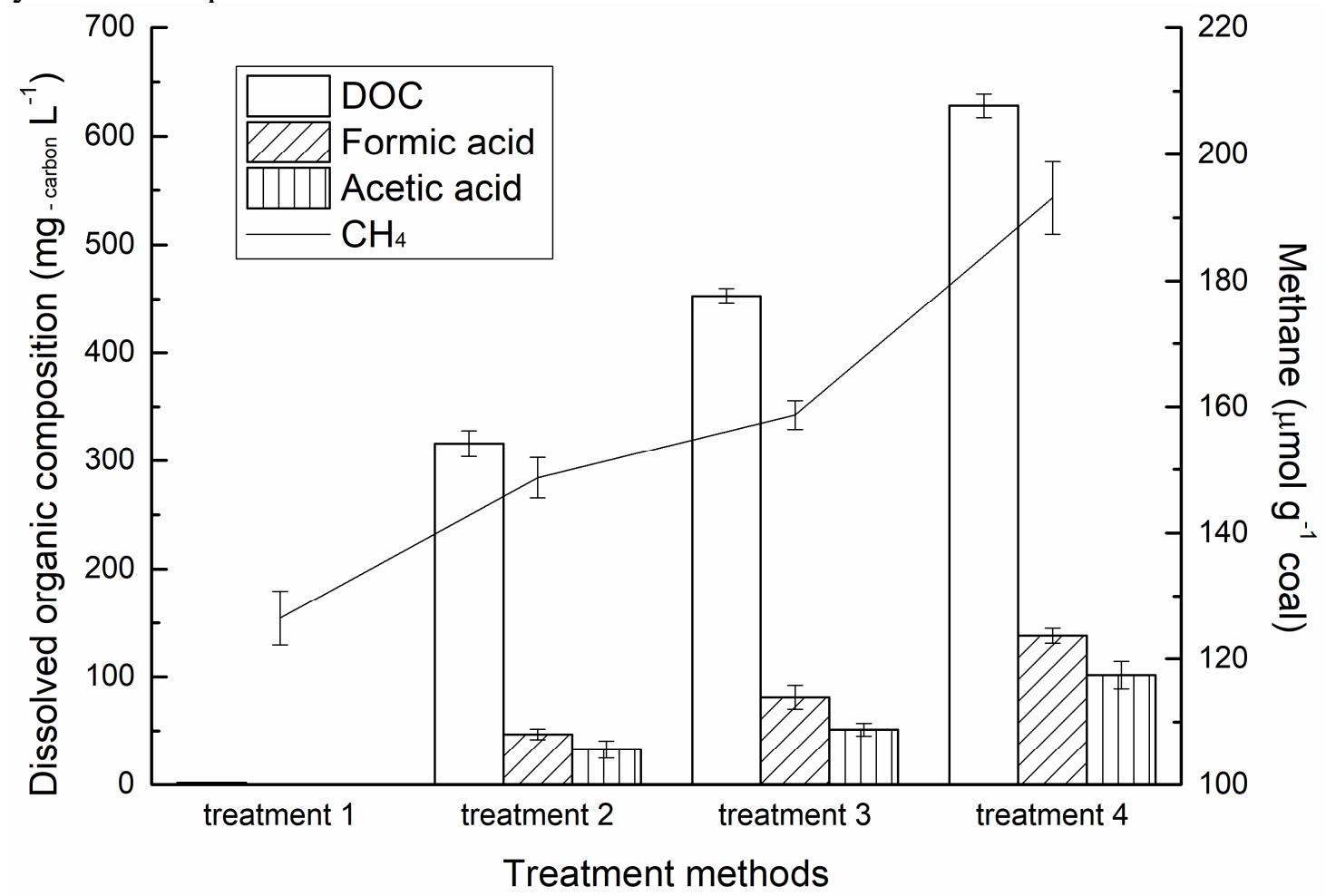

Fig. 1 The dissolved organic carbon, formic acid, and acetic acid concentrations in the supernatants with different pretreatments, and the final methane yield after 60 days of culture. Error bars represent the standard deviations of triplicate cultures.

\section{Conclusions}

This study demonstrated that utilizing hydrogen peroxide to pretreat lignite dramatically increased the concentration of the dissolved organic carbon, which increased the organic acids in the aqueous solution. Under the conditions of this experiment, when $2.5 \mathrm{M}$ hydrogen peroxide was used as the pretreatment method, methane production increased by $53 \%$ compared with untreated lignite. These results illustrated the solubility of coal in water can greatly affect bioavailability.

\section{Acknowledgements}

This work was financially supported by the Tianjin Natural Science Foundation (09JCZDJC26200) and the Tianjin Natural Science Foundation (043611111).

\section{References}

[1] Iram, A., K. Akhtar, and M.A. Ghauri, Coal methanogenesis: a review of the need of complex microbial consortia and culture conditions for the effective bioconversion of coal into methane. Annals of Microbiology, 2017: p. 1-12. 
[2] Gao, Y., et al., Catalytic activity of char produced from brown coal for steam-gasification of bitumen oil. Petroleum Science \& Technology, 2017: p. 1-4.

[3] Chen, T., et al., Characterisation of bioavailability of Surat Basin Walloon coals for biogenic methane production using environmental microbial consortia. International Journal of Coal Geology, 2017. 179.

[4] Yoon, S.P., J.Y. Jeon, and H.S. Lim, Stimulation of biogenic methane generation from lignite through supplying an external substrate. International Journal of Coal Geology, 2016. 162: p. $39-44$.

[5] Tamamura, S., et al., Reaction of lignite with dilute hydrogen peroxide to produce substrates for methanogens at in situ subsurface temperatures. International Journal of Coal Geology, 2016. 167: p. 230-237.

[6] Park, S.Y. and Y. Liang, Biogenic methane production from coal: A review on recent research and development on microbially enhanced coalbed methane (MEcoalbed methane). Fuel, 2016. 166: p. 258-267.

[7] Huang, Z., et al., Low carbon renewable natural gas production from coalbeds and implications for carbon capture and storage. Nature Communications, 2017. 8(1): p. 568.

[8] Huang, Z., M.A. Urynowicz, and P.J.S. Colberg, Stimulation of biogenic methane generation in coal samples following chemical treatment with potassium permanganate. Fuel, 2013. 111(5): p. 813-819.

[9] Hazrin-Chong, N.H., et al., Surface analysis reveals biogenic oxidation of sub-bituminous coal by Pseudomonas fluorescens. Applied Microbiology \& Biotechnology, 2014. 98(14): p. 6443-6452. 